\title{
Article \\ A Tool for Rapid Assessment of Functional Outcomes in Patients with Head and Neck Cancer
}

\author{
Daniel Dejaco ${ }^{1} \oplus$, David Riedl $\left.{ }^{2, *} \mathbb{(}\right)$, Sebastian Gasser ${ }^{1}$, Volker Hans Schartinger ${ }^{1} \oplus$, Veronika Innerhofer ${ }^{1}$, \\ Timo Gottfried ${ }^{1}$, Teresa Bernadette Steinbichler ${ }^{1}$, Felix Riechelmann ${ }^{3}$, Roland Moschen ${ }^{2}$, Oliver Galvan ${ }^{4}$, \\ Robert Stigler ${ }^{5}$, Robert Gassner ${ }^{5}$, Gerhard Rumpold ${ }^{2}$, Anna Lettenbichler-Haug ${ }^{1}$ and Herbert Riechelmann ${ }^{1}$
}

\section{check for}

updates

Citation: Dejaco, D.; Riedl, D.; Gasser, S.; Schartinger, V.H.; Innerhofer, V.; Gottfried, T.; Steinbichler, T.B.; Riechelmann, F.; Moschen, R.; Galvan, O.; et al. A Tool for Rapid Assessment of Functional Outcomes in Patients with Head and Neck Cancer. Cancers 2021, 13, 5529. https://doi.org/10.3390/ cancers 13215529

Academic Editor: Amanda Psyrri

Received: 29 September 2021

Accepted: 3 November 2021

Published: 3 November 2021

Publisher's Note: MDPI stays neutral with regard to jurisdictional claims in published maps and institutional affiliations.

Copyright: (c) 2021 by the authors. Licensee MDPI, Basel, Switzerland. This article is an open access article distributed under the terms and conditions of the Creative Commons Attribution (CC BY) license (https:// creativecommons.org/licenses/by/ $4.0 /$ )
1 Department of Otorhinolaryngology—Head and Neck Surgery, Medical University of Innsbruck, 6020 Innsbruck, Austria; daniel.dejaco@i-med.ac.at (D.D.); sebastian.gasser@student.i-med.ac.at (S.G.); volker.schartinger@i-med.ac.at (V.H.S.); veronika.innerhofer@tirol-kliniken.at (V.I.); Timo.Gottfried@tirol-kliniken.at (T.G.); teresa.steinbichler@tirol-kliniken.at (T.B.S.); praxis@hno-am-dez.at (A.L.-H.); herbert.riechelmann@tirol-kliniken.at (H.R.)

2 Department of Psychiatry, Psychotherapy and Psychosomatics, Medical University of Innsbruck, 6020 Innsbruck, Austria; roland.moschen@tirol-kliniken.at (R.M.); gerhard.rumpold@tirol-kliniken.at (G.R.)

3 Department of Orthopedic Surgery and Traumatology, Medical University of Innsbruck, 6020 Innsbruck, Austria; felix.riechelmann@tirol-kliniken.at

4 Department for Speech, Voice and Swallowing, Medical University of Innsbruck, 6020 Innsbruck, Austria; oliver.galvan@tirol-kliniken.at

5 Department of Oral and Maxillofacial Surgery, Medical University of Innsbruck, 6020 Innsbruck, Austria; robert.stigler@tirol-kliniken.at (R.S.); robert.gassner@tirol-kliniken.at (R.G.)

* Correspondence: david.riedl@tirol-kliniken.at; Tel.: +43-512-504-26-231

Simple Summary: Head and neck cancer and its treatment can lead to various functional impairments. We developed and validated an instrument for rapid physician-rated assessment of basic functional outcomes in HNC patients referred to as "head and neck functional integrity scales" (HNC-FIT scales). Six basic HNC-relevant functions were identified and assigned to verbal ratings based on observable criteria. Face and content validity levels were judged adequate in systematic review by 15 experts. Validity, reliability, and responsiveness were assessed in 37 healthy controls and $84 \mathrm{HNC}$ patients. All domains correlated closely with the outcome of corresponding scales of the reference questionnaire, indicating good construct and criterion validity. For all domains, interrater reliability and retest reliability were $\geq 0.90$ and responsiveness was $\geq 0.15(p<0.01)$. Median completion time for the HNC-FIT scales was $<80 \mathrm{~s}$. Thus, the HNC-FIT scale appeared to be a rapid tool for physician-rated assessment of basic functional outcomes in HNC patients with good validity, reliability, and responsiveness.

Abstract: Head and neck cancer (HNC) and its treatment can lead to various functional impairments. We developed and validated an instrument for rapid physician-rated assessment of basic functional outcomes in HNC patients. HNC-relevant functional domains were identified through a literature review and assigned to verbal ratings based on observable criteria. The instrument draft was subjected to systematic expert review to assess its face and content validity. Finally, the empirical validity, reliability, and responsiveness of the expert-adapted Functional Integrity in Head and Neck Cancer (HNC-FIT) scales were assessed in healthy controls and in HNC patients. A matrix of the 6 functional domains of oral food intake, respiration, speech, pain, mood, and neck and shoulder mobility was created, each with 5 verbal rating levels. Face and content validity levels of the HNCFIT scales were judged to be adequate by 17 experts. In 37 control subjects, 24 patients with HNC before treatment, and in $60 \mathrm{HNC}$ patients after treatment, the HNC-FIT ratings in the 3 groups behaved as expected and functional domains correlated closely with the outcome of corresponding scales of the EORTC-HN35-QoL questionnaire, indicating good construct and criterion validity. Interrater reliability (rICC) was $\geq 0.9$ for all functional domains and retest reliability (rICC) was $\geq 0.93$ for all domains except mood (rICC $=0.71$ ). The treatment effect size (eta-square) as a measure of responsiveness was $\geq 0.15(p<0.01)$ for fall domains except for breathing and neck and shoulder mobility. The median HNC-FIT scale completion time was $1 \mathrm{~min} 17 \mathrm{~s}$. The HNC-FIT scale is a rapid 
tool for physician-rated assessment of functional outcomes in HNC patients with good validity, reliability, and responsiveness.

Keywords: head and neck neoplasms; health status; functional outcomes; surveys and questionnaires; questionnaire design

\section{Introduction}

Recent advances in the diagnosis and treatment of head and neck cancer (HNC) have significantly improved the survival of affected patients and resulted in more long-term survivors [1-3]. Although survival is the most important aspect for HNC patients [4-6], the functional outcome, psychological status and health-related quality-of-life (QoL), social interactions, and economic status are becoming increasingly important as a result of this trend [7]. In a recent survey among head and neck surgeons, more than half reported that they do not systematically record functional outcomes in HNC patients. Obstacles preventing the assessment of functional outcomes included lack of support for recording and analyzing the data. The instruments were too cumbersome for many participants and the time required was too long. Many reported patient difficulties in completing questionnaires or performing tests [8].

This is contrasted by a variety of HNC-specific assessment instruments. Osborn and coauthors list 173 outcome assessment tools for HNC patients, but few are in common use [8]. The National Cancer Institute Common Terminology Criteria for Adverse Events may serve to assess adverse functional outcomes. Verbal rating scales are provided to describe severity for each adverse event term [9]. The Performance Status Scale for Head and Neck Cancer Patients is a clinician-rated instrument consisting of three subscales: normality of diet, understandability of speech, and eating in public [10]. Several HNC-specific QoL questionnaires also include functional ability subscales [8]. The revised University of Washington Head and Neck Quality-of-Life Questionnaire (UW-QoL) is a common QoL instrument in the US. The major parts consist of functional assessment scales [11-13]. The Functional Assessment of Cancer Therapy-Head and Neck Scale [14] is primarily about well-being and not about assessing functional deficits; however, HNC-specific questions also ask about functional aspects. The European Organization for Research and Treatment of Cancer Head and Neck Questionnaire 35 (EORTC QLQ-H\&N35) serves as an HNC-specific QoL questionnaire. It is used together with the basic EORTC QLQC30. The EORTC QLQ-H\&N35 contains 30 questions related to functional outcomes in HNC patients [15]. The most extensive functional assessment tool is the International Classification of Functioning, Disability, and Health (ICF). It provides a description of situations with regard to human functioning and its restrictions and serves as a framework to organize this information [16]. Due to the holistic approach and the high complexity, the ICF is not suitable for routine clinical recording of functional outcome at oncological follow-up visits. Although core sets have been extracted for clinical application in HNC patients [17], to our knowledge there is currently no clinically applicable and formally validated instrument for the assessment of functional outcomes based on the ICF.

The aim of this study was to develop and validate a rapid clinical tool to assess basic physical and mental functional outcomes of HNC patients during routine oncology followup. The tool should be limited to a few relevant HNC-related functional domains and avoid redundancy. Scores should be based on external, observable indicators that can be ranked, with ranks ideally equally distributed across functional domains. Rating scales should be positively aligned so that low scores represent functional limitation, with high scores representing functional integrity. 


\section{Materials and Methods}

The head and neck functional integrity (HNC-FIT) scales was developed in a stepwise approach, as recommended by the Quality-of-Life Group of the EORTC [18]. Since these guidelines were created for the development of patient-reported outcomes, phases were adapted accordingly. The instrument was developed in 4 phases $[18,19]$. In the first phase, functions and symptoms most relevant for HNC patients were identified and condensed into a few functional domains. In the second phase, observable criteria for a uniform scoring system were developed and a draft of the HNC-FIT scales was created. In the third phase, draft HNC-FIT scales were evaluated and adapted by a group of HNC experts from different disciplines and professions. Finally, the adapted HNC-FIT scales were formally validated in healthy controls and HNC patients. The study was approved by the ethics committee of the Medical University of Innsbruck (1182/2019).

\subsection{Identification of HNC-Relevant Functional Domains}

A literature search was performed using the search terms "head and neck cancer", "functional outcome", and "questionnaire" in the National Library of Medicine database. All English-language studies between 1985 and 2020 were reviewed. In addition, commonly used outcome instruments were evaluated. Study titles and abstract were screened for relevance. Studies considered not relevant to the topic were excluded. Exclusion criteria were (a) irrelevant primary tumor site (e.g., esophagus, colorectal, thyroid, parathyroid, skin, lung, bladder, soft-tissue, brain), (b) irrelevant histology (e.g., lymphoma, mucosal melanoma, mesothelioma, retinoblastoma), or (c) other reasons (e.g., pediatric patients, record did not explore cancer, record not in English). Functions and symptoms that occurred in the title, abstract, or full text of multiple publications were identified and assigned to as few higher-level HNC-specific functional domains as possible, such as oral food intake (eating and drinking), breathing, or speech (voice and articulation). Functional domains were chosen to avoid overlap with other functional domains, i.e., to have low redundancy.

\subsection{External Criteria for Uniform Scoring and Draft Development}

Functional integrity was recorded ordinally on verbal rating scales. Verbal ratings ranged from phrases implying complete loss of function or worst functional outcome to normal function, i.e., functional integrity. Normal function was defined as the individual functional status before the onset of disease. As with the UW-QoL and CTCAE approaches, verbal ratings should not reflect the patients' or physicians' subjective impression but should be anchored to external observable criteria to provide some degree of objectivity $[9,13]$. The wording was chosen so that the levels reflected the extent of functional impairment as evenly as possible across all functional domains (equidistance). $\mathrm{Nu}-$ merical scores were assigned to the verbal ratings, ranging from zero for the worst outcome to the highest number for functional integrity (positive scale). To allow rapid completion by ticking by the examining clinician, the functional domains and their verbal ratings were arranged in a matrix. Finally, a draft and instructions on how to complete the HNC-FIT scales were created.

\subsection{Draft Revision through Semi-Structured Expert Interviews}

Face and content validity were assessed by structured interviews with experts in the diagnosis and treatment of HNC patients or experts in one of the identified functional domains. Good face validity was assumed if the mean of the expert rating for global plausibility was less than 2 (good) on a Likert scale of 1 (very good) to 5 (not sufficient). For content validity, each functional domain was assessed with 8 questions on the same 5-point Likert scale. For each of these questions, the scores were recorded, along with the experts comments and suggestions for improvement. If the average of the experts mean score of the 8 questions for a functional domain was above 2, the functional domain was discussed in detail with the experts and corrections were made as necessary. Corrections had to be consistent with the main intention of the instrument to capture functional outcome, 
not well-being. Requested additional functional domains had to occur with sufficient frequency in the performed literature search and had to be recordable in verbal ratings using observable criteria. In a complex coordination process, the adapted version of the HNC-FIT scales was then created.

\subsection{Empirical Validation of the Adapted HNC-FIT Scale}

The adapted HNC-FIT scale was a singly ordered matrix of six functional domains with five levels each. The clinician used this matrix as a template for a structured patient interview and ticked the appropriate functional scores in the matrix.

\subsubsection{Patients and Controls}

HNC patients from the University Department of Otorhinolaryngology-Head and Neck Surgery, Medical University of Innsbruck, Austria, were asked to participate in the validation of the HNC-FIT scales. Inclusion criteria for HNC patients were age $\geq 18$ years and histologically confirmed HNC from the oral cavity, oropharynx, larynx, hypopharynx, or carcinoma of unknown primary with any UICC stage. Patients with cognitive impairment were excluded. Patients with incident HNC before treatment were prospectively recruited in the order of their arrival at the department's outpatient clinic in 2018 and 2019 (pretreatment group). Patients with incident HNC after curative treatment were recruited in the same way during oncology follow-up (posttreatment group). Various clinical characteristics such as age; sex; histology; tumor location; UICC stage; and T-, $\mathrm{N}$-, and M- stages were recorded. Feeding tubes were always placed via percutaneous endoscopic gastrostomy.

Control subjects were approached in the cafeteria of the University Hospital Innsbruck in approximately the known age and sex distribution of HNC patients and asked if they were currently healthy and if they would be willing to participate as controls in this study. The cafeteria is frequented by academic staff, nursing staff, administrative staff, and workers. The age and gender of controls were recorded. Informed consent was obtained from all volunteers.

\subsubsection{Empirical Validity, Reliability, Responsiveness, and Fill-In Time}

Empirical validity was assessed in two ways. For construct validity, it was tested if the HNC-FIT scales behave as expected. Best outcomes were expected in healthy controls, followed by patients with incident HNC before treatment, followed by HNC patients after treatment. To assess this trend, mean ranks of the three participant groups for each functional domain were calculated and tested using the Jonckheere-Terpstra trend test with correction for ties. To assess criterion validity, the EORTC QoL H\&N35 was completed by the HNC patients. The German version of the EORTC H\&N35 was provided by the EORTC according to a license agreement. Spearman's correlation coefficients were calculated between each functional domain and corresponding EORTC H\&N35 subscales and items. While for the HNC-FIT scales for food intake, speech, and pain, clearly corresponding EORTC H\&N35 subscales were available, no corresponding subscales for breathing, mood, or neck and shoulder mobility were available.

Two types of reliability were assessed. To assess interrater reliability, two physicians consecutively completed the adapted HNC-FIT scales in random order, blinded to each other. To assess retest reliability, the HNC-FIT scales were reassessed 5 to 10 days after the last assessment in posttreatment patients by the same rater. For interrater and test-retest reliability in posttreatment patients, intraclass correlation coefficients (ICC) were calculated using a two-way mixed effects, absolute agreement, same raters model.

Responsiveness was assessed in pretreatment patients, since in these patients treatmentrelated changes in functional integrity were to be expected. HNC-FIT scales were completed before treatment and again at the first follow-up visit after the end of treatment (i.e., after surgery only or after multimodality treatment consisting either of primary radiochemotherapy or of surgery followed by postoperative radiation). For responsiveness, we calculated 
a repeated measures ANOVA before and after treatment. Partial eta square as a measure of effects size served to evaluate responsiveness, with values of $\eta 2=0.01, \eta 2=0.06$, and $\eta 2=0.14$ indicating small, medium, and large effects, respectively [20]. Finally, the time to complete the HNC-FIT scales was recorded with a stopwatch.

\subsubsection{Sample Size Estimation}

The sample size estimate for assessment of responsiveness was based on a repeatedmeasures ANOVA with two time-points. The $\alpha$-error was set to 0.05 , the $\beta$-error to 0.2 , $\mathrm{r}$ to 0.5 , and $\mathrm{f}$ to 0.25 , resulting in a sample size of $34 \mathrm{HNC}$ patients for the responsiveness study. For assessment of validity, the sample size for mean differences was calculated with a large effect size with three groups (HNC patients before treatment, after treatment, and controls). An ANOVA with identical parameters as above was assumed for this analysis. This resulted in a total sample size of 64 subjects. Sample size estimates were calculated using GPower3.1 [21]. For sample size estimates of intraclass correlation coefficients, the online sample size calculator provided by Arifin was used [22], assuming an expected reliability of $0.8 \pm 0.15$, an $\alpha$-error of 0.05 , and a power of 0.8 , resulting in a sample size of 24 .

\subsection{Data Analysis}

Frequencies, nominal data, and ordinal data were tabulated. For interval scaled data, means and standard deviations were calculated if not stated otherwise. The scores for each functional domain were dichotomized into normal and near-normal functional outcomes (numerical scores 3 and 4) vs. impaired functional outcomes (numerical scores $0-2$ ). The percentage of patients achieving normal or near-normal functional outcomes (functional integrity) were calculated and depicted in a star plot. For multiple comparisons, p-values were corrected using the Holms-Bonferroni method [23] to avoid risk of type I error. Statistical analyses were performed using SPSS 27 (IBM, Armonk, NY, USA) if not stated otherwise.

\section{Results}

\subsection{HNC-Relevant Functional Domains}

The literature search yielded 1273 records. Of these, 120 articles complied with exclusion criteria and were subjected to full text analysis (Supplemental Data S1), together with the previously identified publications. The full text analysis revealed 39 functions and symptoms, which were initially assigned to 7 functional domains. These were oral food intake, saliva, respiration, speech, pain, mood, and neck-shoulder mobility (Supplemental Data S2). As in the International Classification of Functioning, Disability, and Health, pain and mood were conceived as functions [16].

\subsection{External Criteria for Uniform Scoring}

Observable, external criteria for creating an ordered set of verbal ratings included dependence on a feeding tube and normality of diet for the oral food intake domain, dependence on a tracheotomy and dyspnea for the breathing domain, pain medication for the pain domain, need for antidepressants and feeling depressed for the mood domain, and problems combing hair and looking backward while driving for the neck and shoulder mobility domain. The wording of the verbal rating scales implicating a reasonably uniform ordering of functional integrity allowed five levels of functional integrity for each functional domain (Supplemental Data S3). Despite considerable efforts, we were not able to code the functional domain for dry mouth, chewing, and dental status using external criteria with verbal rating scales in a meaningful sequence (Supplemental Data S2). This functional domain was assigned to the functional domain food intake (Supplemental Data S3). Finally, a draft with a matrix of six functional domains with five levels each was created for expert review (Supplemental Data S4). Detailed instructions on how to interpret items and how to complete this preliminary version of the HNC-FIT scale were provided on the back of 
the form (Supplemental Data S5). HNC-related symptoms and functions, which could not be coded to functional domains or could not be operationalized in a meaningful way, are presented as Supplemental Data S6.

\subsection{Draft Revision through Semi-Structured Expert Interviews}

The draft HNC-FIT scale was reviewed by 17 experts in the multidisciplinary treatment of HNC patients (Supplemental Data S4). Of these, 9 were women. The average length of professional experience in each discipline was $15.7 \pm 5.5$ years. The expert team consisted of 2 otolaryngologists, 2 maxillofacial surgeons, 2 medical oncologists, 1 radiation therapist, 1 orthopedist, 2 phoniatrics, 1 psychologist, 1 anesthesiologist, 1 physical therapist, and 2 speech therapists. The mean score for global acceptance $(1.7 \pm 0.6)$ met the predefined threshold suggesting acceptable face validity. With regard to content validity, experts pointed out that several functional impairments assessed with the HNC-FIT scale can also be caused by conditions other than HNC, e.g., preexisting depression or other causes of chronic pain. Therefore, the remark "due to tumor/treatment" was added to HNC-FIT scales (Supplemental Data S3). Additional domains were suggested by some experts, including social interaction; QoL; sleep quality; aesthetic appearance; and dry mouth, chewing, and dental status domains. In intense discussions, it was found that the suggested additional domains either did not meet the main intention of capturing functional outcomes instead of well-being, did not occur frequently enough in the literature review, or could not be operationalized by verbal ratings anchored to external criteria. Based on expert advice, the wording of verbal rating scales was changed for food intake, speech, pain, mood, and shoulder-neck mobility.

\subsection{Empirical Validation of the Expert Adapted HNC-FIT Scale}

\subsubsection{Patients and Controls}

A total of 37 volunteers who considered themselves healthy (controls), 24 pretreatment HNC patients, and 60 posttreatment HNC patients kindly agreed to participate in the evaluation the HNC-FIT scale.

Of the 24 pretreatment HNC patients, 13 received surgical treatment only and 11 received multimodality treatment. For all pretreatment HNC patients, the mean interval between first and second assessment was 55 ( \pm 35 ; range 15-136) days, if receiving surgery only 29 ( \pm 10 ; range 15-50) days, and if receiving multimodality treatment 85 ( \pm 28 days, range 34-136) days.

Of the 60 posttreatment HNC patients, 8 received no surgery only, 15 received surgery only, and 37 received multimodality treatment. Of these, approximately half of the patients $(n=32)$ received their treatment within the last two years and the other half $(n=28)$ within the last 5 years since inclusion. Since all posttreatment HNC patients were assessed and re-assessed within 5 to 10 days, the mean interval was 9 ( \pm 4 ; range $3-18$ ) days. Patient and disease characteristics are summarized in Table 1.

Table 1. Clinical data for HNC patients and controls.

\begin{tabular}{cccc}
\hline & $\begin{array}{c}\text { Controls } \\
(\boldsymbol{n}=\mathbf{3 7 )}\end{array}$ & $\begin{array}{c}\text { Pretreatment Group } \\
(\boldsymbol{n}=\mathbf{2 4})\end{array}$ & $\begin{array}{c}\text { Posttreatment Group } \\
(\boldsymbol{n}=\mathbf{6 0})\end{array}$ \\
\hline Sex & & & 49 \\
Male & 25 & 21 & 11 \\
Female & 12 & 3 & 3 \\
Age & & 3 & 12 \\
$\leq 50$ & 4 & 7 & 19 \\
$51-60$ & 13 & 7 & 19 \\
$71-80$ & 16 & 4 & 5 \\
\hline
\end{tabular}


Table 1. Cont.

\begin{tabular}{|c|c|c|c|}
\hline & $\begin{array}{l}\text { Controls } \\
(n=37)\end{array}$ & $\begin{array}{l}\text { Pretreatment Group } \\
\qquad(n=24)\end{array}$ & $\begin{array}{c}\text { Posttreatment Group } \\
\qquad(n=60)\end{array}$ \\
\hline \multicolumn{4}{|l|}{ P16 status } \\
\hline Negative & n.a. ${ }^{4}$ & 20 & 49 \\
\hline Positive & n.a. ${ }^{4}$ & 4 & 11 \\
\hline \multicolumn{4}{|l|}{ Tumor site } \\
\hline Oral cavity & n.a. ${ }^{4}$ & 5 & 12 \\
\hline Oropharynx & n.a. ${ }^{4}$ & 6 & 25 \\
\hline Hypopharynx & n.a. ${ }^{4}$ & 4 & 8 \\
\hline Larynx & n.a. ${ }^{4}$ & 8 & 13 \\
\hline $\mathrm{CUP}^{1}$ & n.a. 4 & 1 & 2 \\
\hline \multicolumn{4}{|l|}{ Clinical UICC stage $^{2}$} \\
\hline Stage I & n.a. ${ }^{4}$ & 10 & 13 \\
\hline Stage II & n.a. 4 & 5 & 14 \\
\hline Stage III & n.a. ${ }^{4}$ & 2 & 9 \\
\hline Stage IVa & n.a. 4 & 6 & 21 \\
\hline Stage IVb & n.a. ${ }^{4}$ & 1 & 3 \\
\hline Stage IVc & n.a. ${ }^{4}$ & 0 & 0 \\
\hline \multicolumn{4}{|l|}{ Treatment $^{3}$} \\
\hline No surgery only & n.a. ${ }^{4}$ & 0 & 8 \\
\hline Surgery only & n.a. ${ }^{4}$ & 13 & 15 \\
\hline Multimodality & n.a. ${ }^{4}$ & 11 & 37 \\
\hline
\end{tabular}

${ }^{1}$ CUP: cancer of unknown primary; ${ }^{2}$ UICC: Union for International Cancer Control; ${ }^{3}$ Treatment: no surgery only included primary radiation or primary concurrent radiochemotherapy without any surgical intervention; surgery only includes surgical treatment of HNC without any postoperative radiation or radiochemotherapy, multimodality treatment includes surgery follow by postoperative radiation or radiochemotherapy followed by salvage surgery; ${ }^{4}$ n.a.: not applicable.

\subsubsection{Empirical Validity, Reliability, Responsiveness, and Fill-In Time}

As expected, average ranks of HNC-FIT-scores descended from controls to pretreatment to posttreatment patients (Table 2), supporting the construct validity of the HNCFIT scales.

Table 2. Mean ranks in the Jonckheere-Terpstra tests for 6 functional domains in controls, HNC patients before treatment, and HNC patients after treatment.

\begin{tabular}{ccccc}
\hline $\begin{array}{c}\text { Functional } \\
\text { Domain }\end{array}$ & Controls & $\begin{array}{c}\text { Pretreatment } \\
\text { Group }\end{array}$ & $\begin{array}{c}\text { Posttreatment } \\
\text { Group }\end{array}$ & $\boldsymbol{p}_{\text {-Value }} \mathbf{1}^{\mathbf{2}}$ \\
\hline Food intake & 80.8 & 63.5 & 47.9 & $<0.001$ \\
Breathing & 71.7 & 67.8 & 51.7 & 0.004 \\
Speech & 74.4 & 65.3 & 50.4 & 0.001 \\
Pain & 68.9 & 56.5 & 57.9 & 0.140 \\
Mood & 69.3 & 56.8 & 56.7 & 0.140 \\
Mobility & 66.8 & 69.8 & 53.9 & 0.027 \\
\hline
\end{tabular}

${ }^{1}$ Holm-Bonferroni-corrected $p$-values.

In the pain and mood domains, there was a significant step from controls to pre- and posttreatment patients $(p<0.01)$. Similarly, a significant step from control and pretreatment patients to posttreatment average ranks $(p=0.027)$ was observed in the neck and shoulder mobility domain. HNC-FIT scales correlated with the corresponding EORTC QoL H\&N35 subscales supporting criterion validity. The HNC-FIT scale food intake had the highest correlations with the H\&N35 subscales "feeding tube" $(\mathrm{r}=-0.73, p<0.001)$, "swallowing" $(\mathrm{r}=-0.72, p<0.001)$, and "social eating" $(\mathrm{r}=-0.56, p<0.001)$. For the HNC-FIT scale "speech", the highest correlations were found with the H\&N35 subscale "speech" $(\mathrm{r}=-0.55$, 


$$
\begin{aligned}
& p<0.001) \text {, and for the HNC-FIT scale "pain" with the H\&N35 subscales "pain" }(\mathrm{r}=-0.47, \\
& p<0.001) \text { and "pain killers" }(\mathrm{r}=-0.61, p<0.001 \text {; Table } 3) .
\end{aligned}
$$

Table 3. Spearman correlation coefficients of the clinician-rated HNC-FIT scale and the 18 corresponding patient-rated

\begin{tabular}{|c|c|c|c|c|c|c|c|}
\hline & & \multicolumn{6}{|c|}{ HNC-FIT Scale } \\
\hline & & Food Intake & Breathing & Speech & Pain & Mood & Mobility \\
\hline \multirow{18}{*}{ 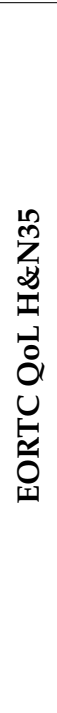 } & Teeth & -0.16 & -0.01 & -0.17 & -0.13 & $-0.32^{3}$ & -0.09 \\
\hline & Swallowing & $-0.72^{1}$ & -0.20 & $-0.31^{3}$ & $-0.31^{3}$ & -0.22 & $-0.41^{2}$ \\
\hline & Social eating & $-0.56^{1}$ & 0.02 & -0.20 & -0.24 & -0.15 & $-0.42^{2}$ \\
\hline & Opening mouth & $-0.36^{2}$ & -0.05 & -0.21 & -0.24 & -0.26 & $-0.40^{2}$ \\
\hline & Dry mouth & $-0.29^{3}$ & 0.15 & 0.10 & -0.12 & -0.06 & -0.21 \\
\hline & Sticky saliva & $-0.38^{2}$ & -0.13 & -0.12 & -0.11 & -0.26 & $-0.32^{3}$ \\
\hline & Nutritional Supplements & $-0.35^{2}$ & -0.02 & -0.09 & -0.15 & -0.09 & -0.07 \\
\hline & Feeding tube & $-0.73^{1}$ & -0.20 & $-0.32^{3}$ & -0.15 & 0.10 & -0.26 \\
\hline & Weight loss & $-0.38^{2}$ & -0.15 & -0.21 & -0.26 & -0.01 & -0.02 \\
\hline & Weight gain & 0.04 & -0.26 & -0.26 & 0.05 & -0.16 & -0.23 \\
\hline & Coughed & -0.15 & -0.16 & -0.15 & 0.03 & -0.11 & 0.05 \\
\hline & Speech & $-0.34^{2}$ & $-0.37^{2}$ & $-0.55^{1}$ & $-0.35^{2}$ & -0.28 & -0.11 \\
\hline & Pain & $-0.36^{2}$ & 0.04 & -0.16 & $-0.47^{1}$ & -0.24 & -0.18 \\
\hline & Pain killers & $-0.35^{2}$ & -0.05 & -0.16 & $-0.61^{1}$ & -0.21 & -0.05 \\
\hline & Social contact & -0.01 & -0.03 & -0.09 & $-0.29^{3}$ & -0.25 & -0.08 \\
\hline & Sexuality & $-0.27^{3}$ & $-0.39^{2}$ & $-0.30^{3}$ & -0.05 & -0.25 & -0.22 \\
\hline & Senses & $-0.37^{2}$ & -0.19 & -0.25 & -0.19 & -0.19 & -0.26 \\
\hline & Feeling ill & $-0.26^{3}$ & -0.14 & -0.09 & -0.11 & $-0.30^{3}$ & -0.21 \\
\hline
\end{tabular}
EORTC QoL H\&N35 subscales in 84 patients with HNC.

${ }^{1} p<0.001 ;{ }^{2} p<0.01 ;{ }^{3} p<0.05$; highest correlations displayed in bold. The negative signs are caused by the opposite scale arrangements. Mobility stands for neck and shoulder mobility. Holm-Bonferroni-corrected $p$-values.

Intraclass correlation coefficients (rICC) for interrater reliability ranged from 0.90 to 0.99 (Table 4). Intraclass correlation coefficients for evaluation of the test-retest reliability between two measurements in posttreatment HNC patients were well above 0.9 for all functional domains except mood, which had an rICC of 0.71 (Table 4).

\begin{tabular}{|c|c|c|c|c|}
\hline & & rICC $^{1}$ & $95 \% \mathrm{CI}^{2}$ & $p$-Value ${ }^{2}$ \\
\hline \multirow{6}{*}{ 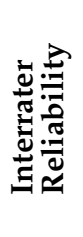 } & Food intake & 0.99 & $0.98-0.99$ & 0.006 \\
\hline & Breathing & 0.95 & $0.93-0.97$ & 0.006 \\
\hline & Speech & 0.96 & $0.94-0.98$ & 0.006 \\
\hline & Pain & 0.93 & $0.89-0.95$ & 0.006 \\
\hline & Mood & 0.91 & $0.86-0.94$ & 0.006 \\
\hline & Shoulder-neck mobility & 0.90 & $0.85-0.93$ & 0.006 \\
\hline \multirow{6}{*}{ 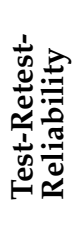 } & Food intake & 0.94 & $0.84-0.98$ & 0.006 \\
\hline & Breathing & 0.95 & $0.91-0.99$ & 0.006 \\
\hline & Speech & 0.97 & $0.94-0.98$ & 0.006 \\
\hline & Pain & 0.93 & $0.81-0.98$ & 0.006 \\
\hline & Mood & 0.71 & $0.18-0.91$ & 0.012 \\
\hline & Dhoulder-neck mobility & 0.98 & $0.93-0.99$ & 0.006 \\
\hline
\end{tabular}

Table 4. Reliability of the adapted HNC-FIT scale.

Note: ${ }^{1} \mathrm{rICC}=$ Intraclass correlation coefficient; ${ }^{2} \mathrm{CI}=$ confidence interval; $\mathrm{rICC}>0.7=$ acceptable, $>0.8=$ good, $>0.9=$ excellent [24]. ${ }^{2}$ Holm-Bonferroni-corrected $p$-values.

To evaluate the responsiveness of the adapted HNC-FIT scale, mean changes before and after treatment for pretreatment HNC patients were compared. The partial eta square, a measure of the effect size in a repeated measures ANOVA before and after treatment, served to evaluate responsiveness. The partial eta square values suggested good responsiveness for food intake $(\eta 2=0.31 ; p=0.040)$ and pain $(\eta 2=0.56 ; p=0.006)$. However, no significant 
responsiveness was observed for breathing $(\eta 2=0.10 ; p=0.33)$, speech $(\eta 2=0.26 ; p=0.08)$, neck and shoulder mobility $(\eta 2=0.01 ; p>0.99)$, or $\operatorname{mood}(\eta 2=0.14 ; p=0.27$; Table 5$)$. The median completion time was $1 \mathrm{~min}$ and $17 \mathrm{~s}$ (25th percentile: $54 \mathrm{~s}$; 75 th percentile: $1 \mathrm{~min}$ and $47 \mathrm{~s}$ ). The shortest completion time was $16 \mathrm{~s}$ and the longest was 3 min and $26 \mathrm{~s}$.

Table 5. Responsiveness values of the HNC-FIT scale.

\begin{tabular}{|c|c|c|c|c|c|}
\hline $\begin{array}{c}\text { Functional } \\
\text { Domain }\end{array}$ & $\begin{array}{l}\text { Pretreatment } \\
\text { Assessment }\end{array}$ & $\begin{array}{l}\text { Posttreatment } \\
\text { Assessment }{ }^{1}\end{array}$ & F-Value & $p$-Value ${ }^{2}$ & $\eta 2$ \\
\hline Food intake & $3.3 \pm 1.0$ & $2.8 \pm 1.1$ & 8.64 & 0.040 & 0.313 \\
\hline Breathing & $3.8 \pm 0.6$ & $3.5 \pm 1$ & 2.11 & 0.326 & 0.100 \\
\hline Speech & $3.8 \pm 0.4$ & $3.2 \pm 1$ & 6.54 & 0.076 & 0.256 \\
\hline Pain & $3.7 \pm 0.8$ & $2.3 \pm 1.1$ & 24.18 & 0.006 & 0.560 \\
\hline Mood & $3.4 \pm 0.8$ & $3.1 \pm 0.9$ & 3.06 & 0.270 & 0.139 \\
\hline Mobility & $3.8 \pm 0.4$ & $3.8 \pm 0.4$ & 0.00 & $>0.99$ & $<0.001$ \\
\hline
\end{tabular}

${ }^{1}$ Mean score \pm standard deviation. ${ }^{2}$ Holm-Bonferroni-corrected $p$-values.

\section{Discussion}

\subsection{Introduction}

Measures of HNC outcomes include survival, HNC-related functional integrity, healthrelated QoL, and economic and social statuses. In a recent survey among head and neck surgeons, more than half reported that they do not systematically record functional outcomes, in part because the instruments are too cumbersome and the time required is too long. In contrast to other common otorhinolaryngologic diseases such as chronic rhinosinusitis [25,26], simple, clinically applicable instruments for recording HNC-related basic physical and mental functions are rare $[8,27]$. The aim of this study was to develop and validate an instrument to assess a basic functional status of HNC patients at oncological follow-up visits. The tool should be able to be applied by the clinician with the least amount of time possible. Only higher-level physiological and mental functional domains such as sight, smell, taste, hearing, pain, mood, food intake, breathing, or speech should be recorded (Supplemental Data S2). Detailed functions and symptoms related to these functional domains were intentionally omitted (Supplemental Data S6). In addition, only functional domains that are commonly affected in HNC patients should be recorded and redundancies should be avoided.

\subsection{Development}

The HNC-FIT scales were developed in 4 phases $[18,19]$. To identify HNC-relevant functional domains, we conducted a literature search on functional outcomes in HNC and initially assigned the most frequently recurring keywords to 7 functional domains. These domains were food intake, saliva, breathing, speech, pain, mood, and shoulder-neck mobility. Other HNC-related functional domains such as vision, hearing, smell, sleep, fatigue, appetite, body image, sexuality, cognitive functioning, anxiety and worry, and social and occupational status did not occur frequently enough in the analyzed publications to be considered. To maintain clarity of the HNC-FIT scales, these domains were not included and should be reserved for more comprehensive functional assessment tools.

The next phase was for operationalization according to the objectives of the study. The initially identified 7 functional domains were arranged in a matrix of similarly graded verbal rating scales from loss of function to normal function. It was found that 5 functional levels could be plausibly formulated into verbal ratings that were linkable to external criteria and had comparable spacing between levels and across functional domains. The dry mouth, chewing, and dental status function could not be operationalized in this way. For "dry mouth", an attempt was made to use the frequency of oral fluid intake due to dry mouth as an external criterion in a pilot study. However, this parameter depended on numerous factors unrelated to saliva production. In addition, this parameter was partially redundant with the functional domain food intake and was omitted. This resulted in a draft 
of 6 verbal rating scales with 5 levels each, for which detailed instructions for clinicians on how to complete it were established. The verbal rating scales were also numerically coded in the sense of a positive 5-point Likert scale from 0 for loss of function or worst outcome to 4 for normal function.

In the third phase of instrument development, this draft was evaluated by 17 experts for face and content validity. Experts were either involved in the multidisciplinary treatment of HNC patients or experts for specific functional domains. While the whole concept of HNC-FIT scales was generally accepted, the experts recommended clarifying whether preexisting, tumor-independent functional limitations should be considered or only functional limitations caused by the HNC. Therefore, the information "due to tumor /treatment" was added to the right of the scales (Supplemental Data S3). Additional functional domains, particularly social function, were suggested by several experts, although corresponding keywords occurred comparatively infrequently in the literature search. Moreover, revision of the functional domains food intake, speech, mood, and shoulder-neck mobility was suggested. Proposed changes were discussed in detail with the experts and adapted to meet the aims of capturing higher-level functional domains rather than specific functions, capturing functional outcomes rather than QoL, and formulating uniformly graded verbal ratings based on external criteria. Several expert suggestions were also included in the instructions for clinicians on how to complete the Head and Neck Functional Integrity Scale (Supplemental Data S4).

\subsection{Mode of Administration}

The mode of administration of outcome assessment instruments may have an impact on the results and data quality [28-30]. We decided against a patient-reported assessment and in favor of a clinician-based assessment. First and foremost, we had concerns about whether patients would be able to correctly understand the verbal ratings. Even physicians needed some explanation and training on how to fill in the HNC-FIT scales. In addition, physician-based assessments have several advantages. The examiner can base their assessment on the medical history, patient interview, and physical examination. In addition, the attending physician can inquire whether anything is unclear or specifically obtain their own assessment of a functional limitation. The clinician-based assessment also ensures that the examiner is aware of the patient's functional limitations and can take appropriate rehabilitative measures. Finally, a certain plausibility check takes place and language barriers or problems with writing or reading can be better resolved.

\subsection{Empirical Validation}

For empirical validation, the adapted HNC-FIT scale (Supplemental Data S3) was evaluated in 37 controls, 24 patients with incident HNC before treatment, and 60 posttreatment HNC patients in oncology follow-up. Controls had approximately the age and sex distribution of HNC patients and considered themselves healthy. It is likely that hospital employees are not representative of the general population of the same age and sex, although no relevant bias was suspected here with respect to the 6 functional domains studied. The included HNC patients were recruited in order of entrance at the clinic and were reasonably representative for HNC patients in our region (Table 1).

To assess the psychometric properties of the adapted HNC-FIT scales, standard techniques were employed $[19,25]$. As expected, there was a descending trend of functional outcomes from controls to pretreatment to posttreatment HNC patients (Table 2). Considering the ordinal level of HNC-FIT scales, mean ranks and the Jonckheere-Terpstra test seemed appropriate for this question. The results of this known-group comparison supported the construct validity of the HNC-FIT scales. The expected trend across the 3 participant groups was highly significant for all domains except mood and pain. However, in the mood domain, controls scored highest $(p<0.01)$ and in the pain domain, posttreatment patients scored lowest $(p<0.01$; Figure 1$)$. 


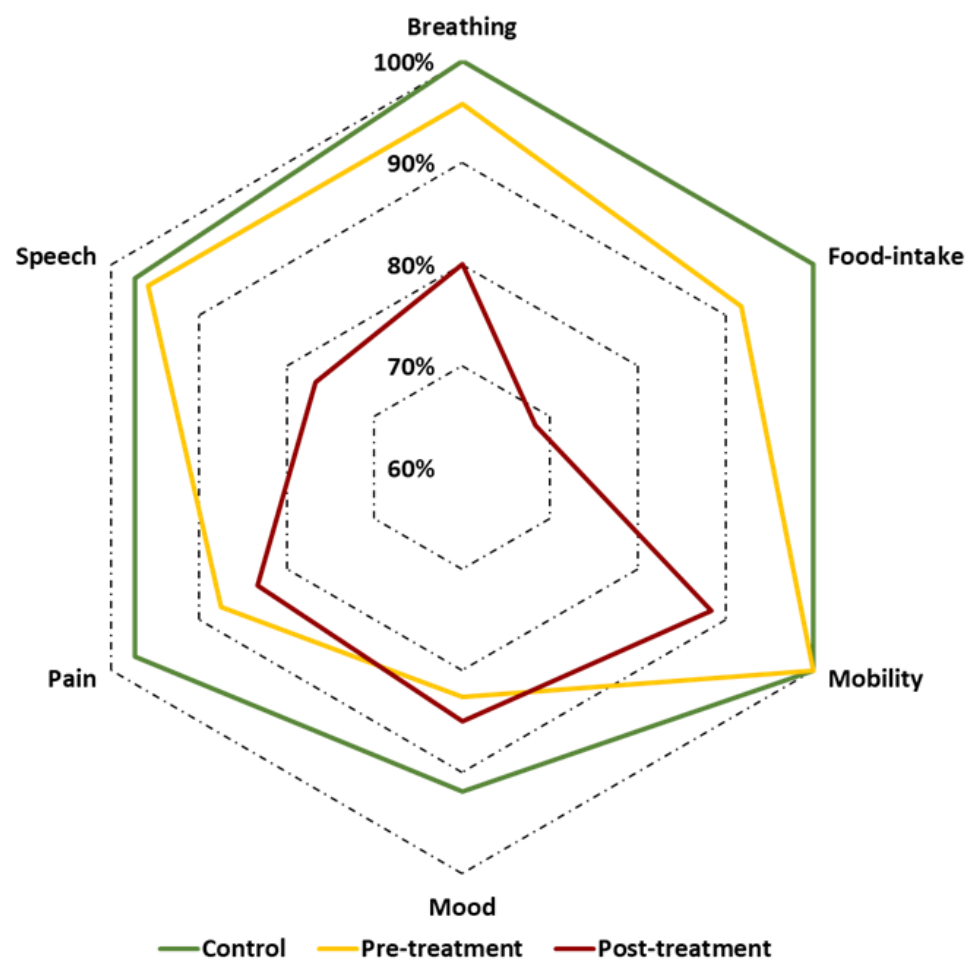

Figure 1. Star plot showing the percentages of normal and near-normal scores (functional integrity; scores 3 and 4) for controls (green line), HNC patients before therapy (yellow line), and HNC patients after therapy (red line). The star axes represent the percentages of study participants with functional integrity for the functional domains breathing, food intake, neck and shoulder mobility, mood, pain, and speech.

It is also reasonable that incident HNC patients do not have a better mood before therapy, i.e., directly after diagnosis, than following therapy. Comparison with the functional scales of the EORTC QoL H\&N35 served to assess criterion validity. Good psychometric properties have been reported for this disease specific QoL questionnaire, which is frequently used in Europe (30). All HNC-FIT scales correlated well with the corresponding patient-reported subscales of the EORTC QoL H\&N35 (Table 3). The reliability of the HNC-FIT scales was estimated with intraclass correlation coefficients (rICC) [19]. Overall, excellent interrater reliability was observed for all functional domains, with rICC scores above 0.9 for all functional domains (Table 4). In addition, excellent values were also observed for retest-reliability for all functional domains [24], except for the functional domain mood, which was in an acceptable range with an rICC of 0.71 (Table 4). Internal consistency (Cronbach's alpha) was not used as a measure of reliability because it implies some redundancy of items [31], which was intentionally avoided. Good responsiveness was observed for the functional domains of food intake, speech, pain, and mood (all $p<0.05)$ (Table 5). Interestingly, this was not observed for respiration $(p=0.17)$ or shoulder-neck mobility $(p=0.67)$. Most patients available for the responsiveness tests had received primary radio- or chemoradiotherapy. Impaired neck and shoulder mobility after radiotherapy often develops within months. This was outside the observation period used to assess responsiveness in this study. In addition, respiratory problems or the need for a tracheostomy are less likely to occur in these patients. Finally, a median completion time of $1 \mathrm{~min}$ and $17 \mathrm{~s}$ for the HNC-FIT scales is considered acceptable, even given the lack of time during oncology follow-up. The 6 score values can be easily entered directly into the clinical information system. 


\subsection{Presentation of Functional Outcomes}

A direct and comprehensive presentation of results is the plain listing of the absolute frequencies with which each verbal rating was marked (Table 6).

Table 6. Frequencies of HNC-FIT verbal ratings.

\begin{tabular}{|c|c|c|c|c|c|}
\hline & Score & Verbal Rating & $\begin{array}{l}\text { Controls } \\
(n=37)\end{array}$ & $\begin{array}{l}\text { Pretreatment } \\
\quad(n=24)\end{array}$ & $\begin{array}{l}\text { Posttreatment } \\
\quad(n=60)\end{array}$ \\
\hline \multirow{5}{*}{ 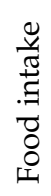 } & 0 & No oral feeding; only via gastrostomy tube & 0 & 0 & 3 \\
\hline & 1 & Gastrostomy tube needed; some oral feeding possible & 0 & 0 & 12 \\
\hline & 2 & No gastrostomy tube, oral diet, but only liquid/soft food & 0 & 2 & 4 \\
\hline & 3 & No gastrostomy tube, diet/swallowing near-normal & 2 & 7 & 15 \\
\hline & 4 & Normal & 35 & 15 & 26 \\
\hline \multirow{5}{*}{ 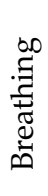 } & 0 & Tracheostoma, needs blocked cannula & 0 & 0 & 2 \\
\hline & 1 & Tracheostoma, speech cannula no cannula & 0 & 1 & 8 \\
\hline & 2 & No tracheostoma, breathing difficult at rest & 0 & 0 & 2 \\
\hline & 3 & No tracheostoma, breathing difficulties only on exertion & 4 & 3 & 13 \\
\hline & 4 & Normal & 33 & 20 & 35 \\
\hline \multirow{5}{*}{$\begin{array}{l}\text { ปू } \\
\text { ญू } \\
\text { की }\end{array}$} & 0 & Not possible/without phonation & 0 & 0 & 4 \\
\hline & 1 & Difficult to understand, no phone calls & 0 & 1 & 5 \\
\hline & 2 & Telephoning possible & 1 & 0 & 5 \\
\hline & 3 & Easy to understand, but pronunciation/voice changed & 3 & 6 & 16 \\
\hline & 4 & Normal & 33 & 17 & 30 \\
\hline \multirow{5}{*}{ ‡ } & 0 & Pain despite opiate therapy & 0 & 0 & 3 \\
\hline & 1 & Controlled with opiates & 0 & 1 & 2 \\
\hline & 2 & Regularly needs non-opioid analgesics & 1 & 2 & 5 \\
\hline & 3 & Needs analgesics from time to time & 2 & 4 & 5 \\
\hline & 4 & Normal & 34 & 17 & 45 \\
\hline \multirow{4}{*}{$\begin{array}{l}0 \\
8 \\
\Sigma\end{array}$} & 0 & Suicidal thoughts & 0 & 0 & 0 \\
\hline & 1 & Very depressed despite antidepressants & 1 & 1 & 3 \\
\hline & 2 & $\begin{array}{l}\text { With antidepressants overall normal mood, very depressed } \\
\text { without antidepressants }\end{array}$ & 2 & 3 & 6 \\
\hline & 3 & Occasionally depressed, no antidepressants needed & 4 & 5 & 15 \\
\hline \multirow{6}{*}{ 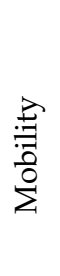 } & 4 & Normal & 30 & 14 & 36 \\
\hline & 0 & Stiff neck and/or shoulder, hardly any movement possible & 0 & 0 & 0 \\
\hline & 1 & $\begin{array}{l}\text { Can hair hairdly comb, looking backwards in car not } \\
\text { possible }\end{array}$ & 0 & 0 & 3 \\
\hline & 2 & Combing with problems, looking backwards in car difficult & 0 & 0 & 4 \\
\hline & 3 & Combing and looking backwards in car slightly restricted & 5 & 2 & 13 \\
\hline & 4 & Normal & 32 & 22 & 40 \\
\hline
\end{tabular}

Relative frequencies are co-determined by the number of participants in each group. Relative frequencies of verbal ratings in percent for the numbers of participants per group can be plotted in a stacked bar chart (Figure 2).

Figure 2 reveals a fairly even distribution of scores across the functional domains within each participant group. This suggests that the formulation of the verbal ratings achieved the goal of uniform scaling across functional domains reasonably well. On the other hand, significant differences are evident between participant groups, which supports the good construct validity. To further ease the outcome interpretation, scores were dichotomized in scores of 0 to 2 (impaired function) vs. 3 and 4 (functional integrity). The outcomes in the control group, where scores $<3$ occurred in only $2.5 \%$, supported this cut-off (Figure 2, left panel). This dichotomization allowed a concise presentation of the basic HNC-relevant functional outcomes in a star graph (Figure 1). It can be easily recognized that for the mood domain, functional integrity was found in only $92 \%$ of controls, whereas it was found in close to $100 \%$ of controls in all other functional domains. Mood, pain, and food intake were the functional domains most frequently impaired in HNC patients before treatment, whereas the other domains were only rarely affected. In posttreatment HNC patients, functional integrity was observed in $80-90 \%$ of patients for 
mood, pain, and neck and shoulder mobility. However, in the speech, breathing, and food intake domains, functional integrity was considerably less frequent. Normal or near-normal food intake was achieved in less than $70 \%$ of posttreatment HNC patients. It is, however, important to consider that these posttreatment results were obtained in a small group of unselected HNC patients for all tumor sites, stages, and treatment modalities. They serve only to demonstrate possible modes of outcome presentation. The sample size was by far too low to draw any general conclusions on functional outcomes in HNC. Mean scores for the 6 functional domains of the 3 study groups are listed in Supplemental Data S7; however, the mean scores depend on the scaling of each specific outcome assessment instrument, are difficult to compare across different instruments, and are considered less intuitive than the percentage of patients achieving normal or near-normal functional outcomes.

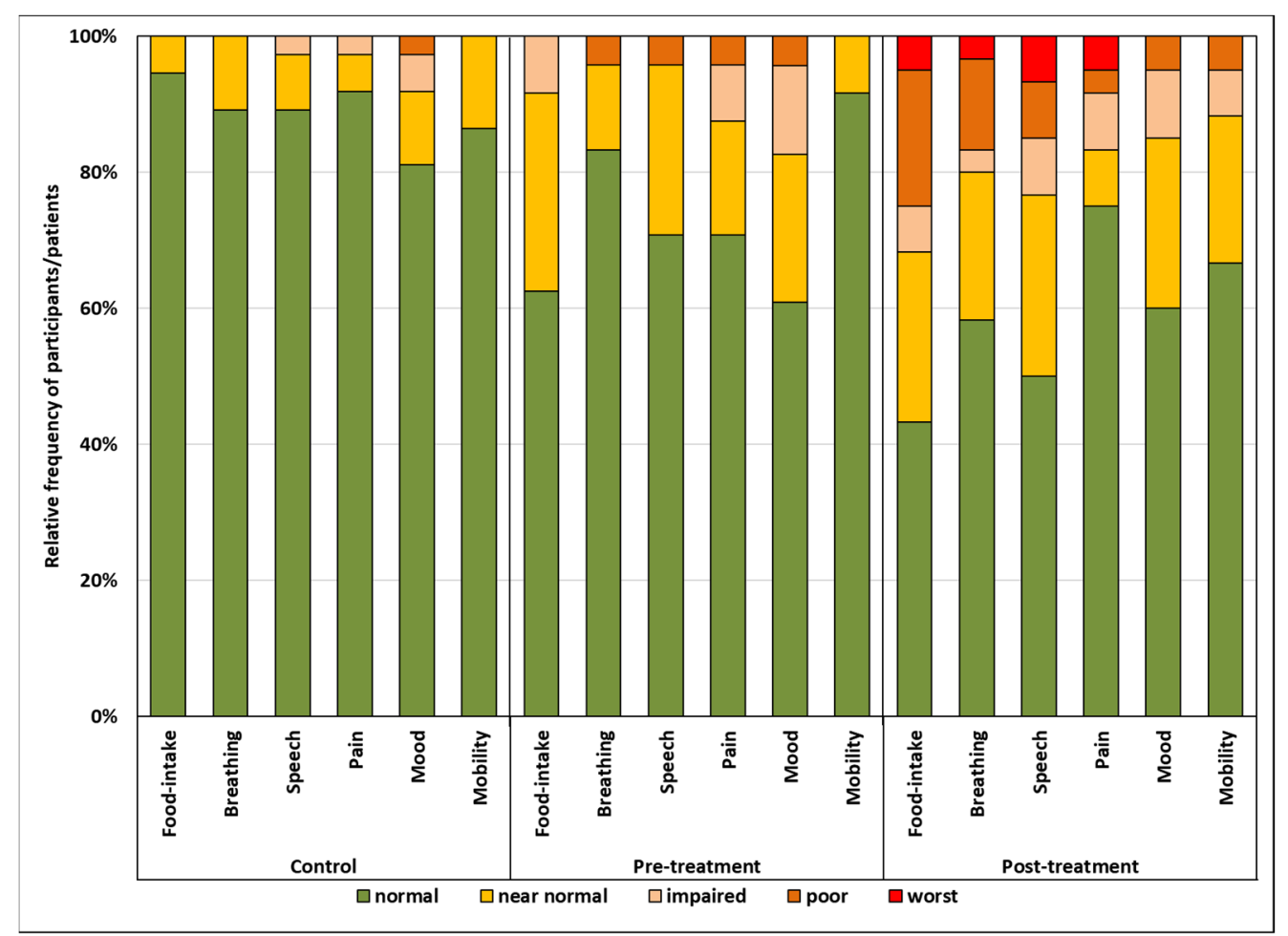

Figure 2. Relative frequencies (percent) of integrity scores in 6 functional domains in controls, HNC patients before treatment, and HNC patients after treatment. Mobility refers to neck and shoulder mobility.

\subsection{Limitations}

The main advantage of the HNC-FIT scales as compact, rapid instruments is also their main limitation. By restricting the scales to the functions and symptoms most frequently mentioned in publications, many important functional domains such as hearing or balance are ignored. This also applies to the assessment of QoL, which was suggested by experts involved in the semi-structured interviews. In intense discussions, it was found that the suggested QoL domain did not meet the main intention of capturing functional outcomes. In addition, it was suggested to include the assessment of dry mouth, chewing, and dental status. Although all three suggestions were considered relevant, despite a considerable effort no meaningful operationalization could be performed (i.e., anchoring to external criteria and equidistance between verbal ratings) (Supplemental Data S6). When functions and symptoms are combined into functional domains for the sake of simplicity, detailed information is certainly lost. This can include dry mouth, chewing, and dental status information, which was subsumed under the higher-level functional domain of oral food intake. The number of functional items and the level of detail are a compromise between the desired characteristics of the outcome assessment instrument and clinical applicability. 
However, various single function assessment tools are available and may supplement the HNC-FIT scales if required (e.g., visual analogic scale to assess QoL, short QoL screeners such as the EQ-5D [32] or the chewing function questionnaire [33]).

As with other outcome assessment instruments, the HNC-FIT scales are subject to various forms of bias [19]. Anchoring assessments to observable external criteria reduces susceptibility to bias. Thus, it can be determined largely without bias whether or not a patient has a tracheostoma or a feeding tube. However, for some items, the investigator relies on the patient's information, which may lead to bias, e.g., due to social desirability. Likely a more relevant source of bias is the halo phenomenon, i.e., that the examiner's overall impression of the patient influences the assessment of individual functional domains. Probably the most important cause of bias in the HNC-FIT scales is that filling them out is cumbersome for both the clinician and the patient and should be done as quickly as possible. The fastest way is to check "normal" for all functional domains. This saves the time needed to check the extent of impairment in detail.

Some additional limitations of the present study ought to be discussed. Firstly, the standardization of the temporal collection of the data was suboptimal. For pretreatment patients, fixed time-points were defined during conceptualization of the study (before treatment and again at the first follow-up visit after end of treatment). However, these intervals significantly varied between patients receiving surgery only (approximately 4 weeks) and patients receiving multimodality treatment (approximately 14 weeks). The latter treatment requires significantly more pretreatment work-up (at our institution usually 4 weeks [34]) and time (usually 6 to 8 weeks) to complete treatment. All pretreatment patients were prospectively recruited in the order of their arrival at the outpatient department. Both the treatment recommendation and consequently the time-point of the first follow-up visit after the end of treatment were based on the recommendation of the institutional interdisciplinary tumor board. Thus, no further optimization of temporal data collection could be achieved. For posttreatment patients, the interval between the first and second assessment was relatively constant at 5 to 10 days. However, the time of assessment during oncologic follow up was not fixed. Therefore, half of the patients were assessed within the first two years and the other half within the third to fifth years after end of treatment. It is probable that functional integrity scores raised with the HNC-FIT scales are influenced by the time-point of assessment during oncologic follow-up. Unfortunately, the number of patients included in this study was too small to observe a significant difference within the posttreatment group $(p>0.12)$.

Secondly, it is also likely that the stage, type of therapy and tumor site influence functional integrity scores raised with the HNC-FIT scales. Unfortunately, the number of patients included in this study was too small to conduct multivariable analyses to investigate the unique contribution of each variable on the total score or domain scores.

Thirdly, the HNC-FIT scales were developed and empirically validated for Germanspeaking patients only. Although the original German versions of the HNC-FIT scales were translated to English by the authors themselves (Supplemental Data S3), the translated HNC-FIT scales had neither been professionally translated nor empirically validated for English-speaking patients. The original German versions of the HNC-FIT scales are provided as supplemental data (Supplemental Data S8).

\section{Conclusions}

The HNC-FIT scale is a plain tool for rapid assessment functional outcomes in HNC patients with good psychometric properties. It allows for quick capture and clear presentation of key functional results, filling a gap in HNC outcome assessment.

Supplementary Materials: The following are available online at https: / www.mdpi.com/article / 10.3390/cancers13215529/s1, Supplemental Data S1: Literature search of 120 included articles. Supplemental Data S2: Mapping of functions or symptoms repetitively mentioned in publications on functional outcome in HNC patients to functional domains. Supplemental Data S3: Adapted Head and Neck Cancer Functional Integrity Scale (HNC-FIT Scale). Supplemental Data S4: Assessment of 
face and content validity of the Head and Neck Cancer Functional Integrity Scale (HNC-FIT scales) via semi-structured expert interviews. Supplemental Data S5: Instructions for clinicians on how to complete the Head and Neck Functional Integrity Scale. Supplemental Data S6: Selection of HNC-related symptoms and functions not covered by HNC-FIT scales. Supplemental Data S7: Mean scores and standard deviations of the adapted HNC-FIT scales. Supplemental Data S8: German version of the adapted Head and Neck Cancer Functional Integrity Scale (HNC-FIT Scale).

Author Contributions: Conceptualization, D.D., D.R. and H.R.; methodology, D.R., S.G. and V.H.S.; validation, S.G., V.I., T.G., T.B.S., F.R., R.M., O.G., R.S., R.G. and G.R.; formal analysis, A.L.-H., D.R. and H.R.; investigation, D.D., S.G., V.I., T.G., T.B.S., F.R., R.M., R.S., R.G. and O.G.; data curation, D.D., D.R. and H.R.; writing—original draft preparation, D.D., D.R. and H.R.; writing-review and editing, D.D., D.R., G.R. and H.R.; visualization, D.D., D.R. and H.R.; supervision, G.R. and H.R. All authors have read and agreed to the published version of the manuscript.

Funding: This research received no external funding.

Institutional Review Board Statement: The study was conducted according to the guidelines of the Declaration of Helsinki and approved by the Institutional Ethics Committee of the Medical University of Innsbruck, Austria (protocol code 1182/2019 and 28 May 2020).

Informed Consent Statement: Informed consent was obtained from all subjects involved in the study.

Data Availability Statement: The data presented in this study are available on request from the corresponding author.

Conflicts of Interest: The authors declare no conflict of interest.

\section{References}

1. Chow, L.Q.M. Head and neck cancer. N. Engl. J. Med. 2020, 382, 60-72. [CrossRef] [PubMed]

2. Johnson, D.E.; Burtness, B.; Leemans, C.R.; Lui, V.W.Y.; Bauman, J.E.; Grandis, J.R. Head and neck squamous cell carcinoma. Nat. Rev. Dis. Primers 2020, 6, 92. [CrossRef] [PubMed]

3. León, X.; Orús, C.; Casasayas, M.; Neumann, E.; Holgado, A.; Quer, M. Trends in disease-specific survival of head and neck squamous cell carcinoma patients treated in a single in-stitution over a 30-year period. Oral Oncol. 2021, 115, 105184. [CrossRef]

4. Brotherston, D.C.; Poon, I.; Le, T.; Leung, M.; Kiss, A.; Ringash, J.; Balogh, J.; Lee, J.; Wright, J.R. Patient preferences for oropharyngeal cancer treatment de-escalation. Head Neck 2012, 35, 151-159. [CrossRef] [PubMed]

5. List, M.A.; Rutherford, J.L.; Stracks, J.; Pauloski, B.R.; Logemann, J.A.; Lundy, D.; Sullivan, P.; Goodwin, W.; Kies, M.; Vokes, E.E. Prioritizing treatment outcomes: Head and neck cancer patients versus nonpatients. Head Neck 2004, 26, 163-170. [CrossRef] [PubMed]

6. List, M.A.; Stracks, J.; Colangelo, L.; Butler, P.; Ganzenko, N.; Lundy, D.; Sullivan, P.; Haraf, D.; Kies, M.; Goodwin, W.; et al. How Do Head and Neck Cancer Patients Prioritize Treatment Outcomes Before Initiating Treatment? J. Clin. Oncol. 2000, 18, 877. [CrossRef]

7. Ringash, J.; Bernstein, L.; Devins, G.; Dunphy, C.; Giuliani, M.; Martino, R.; McEwen, S. Head and Neck Cancer Survivorship: Learning the Needs, Meeting the Needs. Semin. Radiat. Oncol. 2018, 28, 64-74. [CrossRef] [PubMed]

8. Osborn, H.A.; Ma, T.A.G.; Varvares, M.A. Assessing functional outcomes in head and neck surgical oncology. Head Neck 2019, 41, 2051-2057. [CrossRef]

9. National Cancer Institute. Common Terminology Criteria for Adverse Events (CTCAE). 2017; p. 155. Available online: https:/ / ctep.cancer.gov/protocoldevelopment/electronic_applications/ctc.htm (accessed on 3 November 2021).

10. List, M.A.; D'Antonio, L.L.; Cella, D.F.; Siston, A.; Mumby, P.; Haraf, D.; Vokes, E. The performance status scale for head and neck cancer patients and the functional assessment of cancer therapy-head and neck scale: A study of utility and validity. Cancer 1996, 77, 2294-2301. [CrossRef]

11. Weymuller, E.A., Jr.; Alsarraf, R.; Yueh, B.; Deleyiannis, F.W.B.; Coltrera, M.D. Analysis of the performance characteristics of the University of Washington Quality of Life in-strument and its modification (UW-QOL-R). Arch. Otolaryngol. Head Neck Surg. 2001, 127, 489-493. [CrossRef] [PubMed]

12. Rogers, S.N.; Gwanne, S.; Lowe, D.; Humphris, G.; Yueh, B.; Weymuller, E.A., Jr. The addition of mood and anxiety domains to the University of Washington quality of life scale. Head Neck 2002, 24, 521-529. [CrossRef]

13. Rogers, S.N.; Lowe, D.; Yueh, B.; Weymuller, E.A., Jr. The Physical Function and Social-Emotional Function Subscales of the University of Washington Quality of Life Questionnaire. Arch. Otolaryngol. Head Neck Surg. 2010, 136, 352-357. [CrossRef]

14. D'Antonio, L.L.; Zimmerman, G.J.; Cella, D.F.; Long, S.A. Quality of Life and Functional Status Measures in Patients with Head and Neck Cancer. Arch. Otolaryngol. Head Neck Surg. 1996, 122, 482-487. [CrossRef] [PubMed] 
15. Bjordal, K.; De Graeff, A.; Fayers, P.M.; Hammerlid, E.; van Pottelsberghe, C.; Curran, D.; Ahlner-Elmqvist, M.; Maher, E.J.; Meyza, J.W.; Bredart, A.; et al. A 12 country field study of the EORTC QLQ-C30 (version 3.0) and the head and neck cancer specific mod-ule (EORTC QLQ-H\&N35) in head and neck patients. EORTC Quality of Life Group. Eur. J. Cancer 2000, 36, $1796-1807$. [PubMed]

16. World Health Organization. International Classification of Functioning, Disability and Health: ICF; World Health Organization: Geneva, Switzerland, 2019.

17. Kisser, U.; Adderson-Kisser, C.; Coenen, M.; Stier-Jarmer, M.; Becker, S.; Sabariego, C.; Harréus, U. The development of an ICF-based clinical guideline and screening tool for the standardized assessment and evaluation of functioning after head and neck cancer treatment. Eur. Arch. Oto-Rhino-Laryngol. 2016, 274, 1035-1043. [CrossRef] [PubMed]

18. Johnson, C.; Aaronson, N.; Blazeby, J.M.; Bottomley, A.; Fayers, P.; Koller, M.; Kuliś, D.; Ramage, J.; Sprangers, M.; Velikova, G.; et al. Guidelines for Developing Questionnaire Modules: EORTC Quality of Life Group. 2011. Available online: https: //www.eortc.org/app/uploads/sites/2/2018/02/guidelines_for_developing_questionnaire-_final.pdf (accessed on 3 November 2021).

19. Streiner, D.L.; Norman, G.R.; Cairney, J. Health Measurement Scales: A Practical Guide to Their Development and Use, 5th ed.; Oxford University Press: Oxford, UK, 2015.

20. Cohen, J. Statistical Power Analysis for the Behavioral Sciences, 2nd ed.; L. Erlbaum Associates: Hillsdale, NJ, USA, 1988.

21. Faul, F.; Erdfelder, E.; Buchner, A.; Lang, A.-G. Statistical power analyses using G*Power 3.1: Tests for correlation and regression analyses. Behav. Res. Methods 2009, 41, 1149-1160. [CrossRef]

22. Arifin, W.N. Sample Size Calculator. 2021. Available online: http:/ / wnarifin.github.io (accessed on 29 September 2021).

23. Holm, S. A simple sequential rejective method procedure. Scand. J. Stat. 1979, 6, 65-70.

24. Portney, L.G.; Watkins, M.P. Foundations of Clinical Research: Applications to Practice, 3rd ed.; Prentice Hall: Hoboken, NJ, USA, 2009.

25. Riedl, D.; Dejaco, D.; Steinbichler, T.; Innerhofer, V.; Gottfried, T.; Bektic-Tadic, L.; Giotakis, A.; Rumpold, G.; Riechelmann, H. Assessment of health-related quality-of-life in patients with chronic Rhinosinusitis-Validation of the German Sino-Nasal Outcome Test-22 (German-SNOT-22). J. Psychosom. Res. 2021, 140, 110316. [CrossRef] [PubMed]

26. Dejaco, D.; Riedl, D.; Huber, A.; Moschen, R.; Giotakis, A.I.; Bektic-Tadic, L.; Steinbichler, T.; Kahler, P.; Riechelmann, H. The SNOT-22 factorial structure in European patients with chronic rhinosinusitis: New clinical insights. Eur. Arch. Oto-Rhino-Laryngol. 2019, 276, 1355-1365. [CrossRef]

27. Mendez, A.; Seikaly, H.; Eurich, D.; Dzioba, A.; Aalto, D.; Osswald, M.; Harris, J.R.; O'Connell, D.A.; Lazarus, C.; Urken, M.; et al. Development of a Patient-Centered Functional Outcomes Questionnaire in Head and Neck Cancer. JAMA Otolaryngol. Neck Surg. 2020, 146, 437. [CrossRef]

28. Bowling, A. Mode of questionnaire administration can have serious effects on data quality. J. Public Health 2005, $27,281-291$. [CrossRef] [PubMed]

29. Cheung, Y.B.; Goh, C.; Thumboo, J.; Khoo, K.-S.; Wee, J. Quality of life scores differed according to mode of administration in a review of three major oncology questionnaires. J. Clin. Epidemiol. 2006, 59, 185-191. [CrossRef]

30. Kirsh, E.; Naunheim, M.; Ma, C.T.G.; Kammer, R.; Varvares, M.; Goldsmith, T. Patient-reported versus physiologic swallowing outcomes in patients with head and neck cancer after chemoradiation. Laryngoscope 2019, 129, 2059-2064. [CrossRef] [PubMed]

31. Boyle, G.J. Does item homogeneity indicate internal consistency or item redundancy in psychometric scales? Pers. Individ. Differ. 1991, 12, 291-294. [CrossRef]

32. Beck, A.C.C.; Kieffer, J.M.; Retèl, V.P.; van Overveld, L.F.; Takes, R.P.; van den Brekel, M.W.; van Harten, W.H.; Stuiver, M.M. Mapping the EORTC QLQ-C30 and QLQ-H\&N35 to the EQ-5D for head and neck cancer: Can dis-ease-specific utilities be obtained? PLoS ONE 2019, 14, e0226077.

33. Baba, K.; John, M.T.; Inukai, M.; Aridome, K.; Igarahsi, Y. Validating an alternate version of the chewing function questionnaire in partially dentate patients. BMC Oral Health 2009, 9, 9. [CrossRef]

34. Dejaco, D.; Steinbichler, T.; Schartinger, V.H.; Fischer, N.; Anegg, M.; Dudas, J.; Posch, A.; Widmann, G.; Riechelmann, H. Specific growth rates calculated from $\mathrm{CT}$ in patients with head and neck squamous cell carcinoma: A retrospective study performed in Austria. BMJ Open 2019, 9, e025359. [CrossRef] [PubMed] 